\title{
Niemand zahlt mehr Miete!? \\ - Die Corona-Krise und ihre Auswirkungen auf die Pflicht zur Mietzahlung
}

\author{
Dr. Jonas David Brinkmann, Bielefeld"
}

\section{Inhaltsübersicht}

$\begin{array}{ll}\text { A. Einleitung } & 147\end{array}$

B. Der neue Art. $240 \$ 2$ EGBGB 149

I. Ratio legis des Art. $240 \$ 2$ EGBGB 150

II. Bloße Verlagerung von Liquiditätsproblemen 152

III. Voraussetzungen des Kündigungsausschlusses 153

1. „Subjektive Unmöglichkeit“ der Zahlung 154

2. Keine Vorprozessuale Pflicht zur Glaubhaftmachung 155

3. Kein Kündigungsausschluss bei Zahlungsverzug vor oder nach dem Krisenzeitraum 156

4. Stichtagsregelung in Art. $240 \$ 2$ Abs. 4 EGBGB 158

5. Umgehungsgefahr durch „Kautionsauffüllungsanspruch“ 160

6. Zwischenfazit 161

C. Auswirkungen der „Corona-Krise“ nach den allgemeinen Regeln des Zivilrechts 161

I. Anwendbarkeit der allgemeinen Regeln des Zivilrechts? 161

II. Mietminderung nach $₫ 536$ Abs. 1 BGB 163

III. Wegfall der Leistungspflicht nach $\$ \$ 326,275$ BGB 169

IV. Störung der Geschäftsgrundlage nach $₫ 313$ BGB 173

D. Fazit 174

\section{A. Einleitung}

„Handel, Hotels, Restaurants - niemand zahlt mehr Miete“ so lautete der Titel eines Beitrags, der am 28. März 2020 auf „Manager-Magazin.de“ veröffentlicht wurde. ${ }^{1}$ Aufgrund der weitgehenden Stilllegung des öffentli-

* Der Verfasser ist akademischer Rat a.Z. und Habilitand an der Universität Bielefeld.

1 Abrufbar unter: www.manager-magazin.de/politik/artikel/corona-krise-handel-hote ls-restaurants-niemand-zahlt-mehr-miete-a-1305795.html (zuletzt abgerufen am 21.04.2020). 
chen Lebens durch die Corona-Krise, heißt es dort, hätten etwa die in vielen Innenstädten präsenten Unternehmen H\&M, Adidas, Deichmann, Christ, Saturn und Mediamarkt beabsichtigt, ab April keine Miete mehr für ihre geschlossenen Standorte zu überweisen. ${ }^{2}$ Auch viele weitere Unternehmen dürften entsprechende Überlegungen angestellt haben bzw. anstellen.

Zur Begründung eines solchen Zahlungsstopps wird mitunter auf das neue sog. "Corona-Rettungsgesetz" ${ }^{3}$ verwiesen. ${ }^{4}$ Bundesjustizministerin Christine Lambrecht, die den Zahlungsstopp finanzstarker Unternehmen als „unanständig und nicht akzeptabel“ geißelte, erklärte, die „Corona-Hilfsgesetze böten dafür keine Grundlage“. ${ }^{5}$ Zwar ist Adidas unterdessen wieder zurückgerudert und hat die Miete für April wohl überwiesen. Gleichwohl stellt sich - abgesehen von der moralischen Bewertung eines Zahlungsstopps als anständig oder unanständig - auch aus juristischer Perspektive die Frage, wer nun Recht mit seiner Einschätzung hat - Frau Lambrecht oder die Mieter, die ihre Miete nicht zahlen wollen? Dieser Frage, die auch bereits Drygala in einem Beitrag in der LTO aufgeworfen hat, ${ }^{6}$ soll im Folgenden genauer nachgegangen werden. Hierzu ist zunächst zu klären, ob die einschlägige Regelung des Corona-Rettungsgesetzes, nämlich der neue Art. $240 \$ 2$ EGBGB, als Rechtfertigung dafür herangezogen werden kann, dass etwa ein Konzern wie Adidas mit einer Netto-Cash-Position von ca. 873 Mio. $€$ (Stand Ende 2019), seinen vertraglichen Pflichten zur Mietzahlung nicht nachkommt (hierzu unter B.). Darüber hinaus gilt es zu bestimmen, welche weiteren Gründe es aus Sicht des Mieters geben könnte, die Entrichtung der vereinbarten Miete vollständig oder teilweise zu verweigern - ob also die angekündigten Zahlungsverweigerungen tatsächlich „nicht akzeptabel“ sind ( hierzu unter C.).

2 Abrufbar unter: https://www.manager-magazin.de/politik/artikel/corona-krise-han del-hotels-restaurants-niemandzahlt-mehr-miete-a-1305795.html (zuletzt abgerufen am 21.04.2020).

3 Gesetz zur Abmilderung der Folgen der COVID-19-Pandemie im Zivil-, Insolvenzund Strafverfahrensrecht, BT-Drucks. 19/18110.

4 https://www.manager-magazin.de/politik/artikel/corona-krise-handel-hotels-restaur ants-niemandzahlt-mehr-miete-a-1305795.html (zuletzt abgerufen am 21.04.2020).

5 www.rnd.de/politik/adidas-und-hm-wollen-keine-miete-mehr-zahlen-lambrecht-em port-YOQLJASEAYCMR7E6EYXZ7YMLR4.html (zuletzt abgerufen am 21.04.2020).

6 T. Drygala, Corona und ausbleibende Gewerbemieten: Adidas handelt juristisch vertretbar, in: Legal Tribune Online, 30.03.2020, abrufbar unter: www.lto.de/persis tent/a_id/41145/ (zuletzt abgerufen am 21.04.2020). 


\section{B. Der neue Art. $240 \$ 2$ EGBGB}

Am 1. April 2020 ist Art. 5 des Gesetzes zur Abmilderung der Folgen der COVID-19-Pandemie im Zivil-, Insolvenz- und Strafverfahrensrecht in Kraft getreten. ${ }^{7}$ Durch die Bestimmung wurde ein neuer Art. 240 in das EGBGB eingeführt, der vertragsrechtliche Regelungen aus Anlass der COVID-19-Pandemie beinhaltet. Neben einem allgemeinen Moratorium für Zahlungspflichten von Verbrauchern und Kleinstunternehmern aus bestimmten Dauerschuldverhältnissen in Art. $240 \$ 1$ EGBGB sieht Art. 240 $\$ 2$ EGBGB spezielle Regelungen zur Kündigung von Miet- und Pachtverhältnissen vor. Bereits ein kurzer Blick ins Gesetz genügt um festzustellen: Eins-zu-Null für Frau Lambrecht. Das Corona-Rettungsgesetz bietet keine Grundlage dafür, dass finanzstarke Unternehmen ihre Miete nicht zahlen. Die Regelung in Art. $240 \$ 2$ EGBGB lautet wie folgt:

$\$ 2$ Beschränkung der Kündigung von Miet- und Pachtverhältnissen

(1) Der Vermieter kann ein Mietverhältnis über Grundstücke oder über Räume nicht allein aus dem Grund kündigen, dass der Mieter im Zeitraum vom 1. April 2020 bis 30. Juni 2020 trotz Fälligkeit die Miete nicht leistet, sofern die Nichtleistung auf den Auswirkungen der COVID-19-Pandemie beruht. Der Zusammenhang zwischen COVID-19Pandemie und Nichtleistung ist glaubhaft zu machen. Sonstige Kündigungsrechte bleiben unberührt.

(2) Von Absatz 1 kann nicht zum Nachteil des Mieters abgewichen werden.

(3) Die Absätze 1 und 2 sind auf Pachtverhältnisse entsprechend anzuwenden.

(4) Die Absätze 1 bis 3 sind nur bis zum 30. Juni 2022 anzuwenden.

Ein Leistungsverweigerungsrecht des Mieters begründet die Vorschrift bereits ihrem Wortlaut nach - anders als die allgemeine Regelung in $\$ 1$ - gerade nicht. Das Leistungsverweigerungsrecht aus Art. $240 \rrbracket 1$ Abs. 1 bzw. Abs. 2 EGBGB soll nach Art. $240 \$ 1$ Abs. 4 Nr. 1 EGBGB nicht für Mietund Pachtverhältnisse gelten. Bei Miet- und Pachtverträgen wird also lediglich das Kündigungsrecht des Vermieters wegen Zahlungsverzug eingeschränkt. Das bestätigt auch die Gesetzesbegründung, die ausdrücklich feststellt: „Mieter erhalten kein Leistungsverweigerungsrecht nach der Grundregel des $₫ 1$. Sie bleiben damit nach allgemeinen Grundsätzen zur Leistung verpflichtet und können gegebenenfalls auch in Verzug gera-

7 Art. 6 Abs. 5 Corona-Rettungsgesetz. 
ten“8. Die Pflicht des Mieters zur Zahlung der Miete bleibt also grds. uneingeschränkt bestehen.

\section{Ratio legis des Art. $240 \$ 2$ EGBGB}

Der Sinn und Zweck der Regelung liegt, wie in der Gesetzesbegründung ausgeführt wird, darin, „dem Interesse am Fortbestand des Mietverhältnisses den Vorzug" "9 vor einem etwaigen Lösungsinteresse des Vermieters zu geben.

Das Bestandsinteresse des Mieters ist grds. auch von einigem Gewicht: Die Wohnung ist für den Mieter der „Lebensmittelpunkt menschlichen Daseins"10. Für den Gewerbetreibenden sind die angemieteten Räume Grundlage ihrer Erwerbstätigkeit. ${ }^{11}$ Dementsprechend ist der vertragstreue Mieter nach dem deutschen Mietrecht in aller Regel auch gut vor Kündigungen des Vermieters geschützt: Im Wohnraummietrecht ist die Möglichkeit des Vermieters zur ordentlichen Kündigung gem. $\$ 573$ BGB auf enge Ausnahmefälle eines berechtigten Interesses begrenzt. In der Gewerberaummiete werden in den überwiegenden Fällen Zeitmietverträge abgeschlossen, bei denen eine ordentliche Kündigung ausgeschlossen ist. ${ }^{12}$ Kommt der Mieter hingegen seiner Verpflichtung zur Mietzahlung nicht nach, kann es allerdings grds. recht schnell zur Kündigung durch den Vermieter kommen: Nach $\$ 543$ Abs. 2 S. 1 Nr. 3 lit. a) BGB ist der Vermieter bereits zur fristlosen außerordentlichen Kündigung berechtigt, wenn der Mieter für zwei aufeinander folgende Termine mit der Entrichtung der Miete oder eines nicht unerheblichen Teils der Miete in Verzug ist. Entsprechendes gilt nach $\$ 573$ BGB mit Blick auf die ordentliche Kündigung des Vermieters: Hier reicht nach der Rspr. und h.M. schon der Verzug mit über einer Monatsmiete für mindestens einen Monat. ${ }^{13}$

8 BT-Drucks. 19/18110, S. 36.

9 BT-Drucks. 19/18110, S. 36.

10 M. Häublein, in: Münchener Kommentar zum BGB, 8. Auflage, München 2020, $\$ 573$ Rn. 1.

11 BT-Drucks. 19/18110, S. 36.

12 I. Hörndler, in: Beck-online.GROSSKOMMENTAR, München, Stand 01.04.2020, $\llbracket 578$ BGB Rn. 66, 70; K. Lützenkirchen, Auswirkungen der Corona-Pandemie auf Mietverhältnisse, MietRB 2020, 1 (3).

13 BGH NJW 2013, 159; U. Börstinghaus, Besonderheiten des Miet- und Wohnungseigentumsrechts infolge der COVID-19-Pandemie, ZAP 2020, 411. 
Die wirtschaftlichen Auswirkungen der SARS-CoV-2-Pandemie drohten deshalb den Verlust des Lebensmittelpunkts bzw. der Erwerbstätigkeitsgrundlage für eine erhebliche Anzahl von Personen zur Folge zu haben. Auf Grund der behördlich angeordneten Schließung einer Vielzahl von Betrieben wie etwa Restaurants oder Einzelhandelsgeschäften und auch wegen des Einbruchs der Aktivität des produzierenden Gewerbes sind erhebliche Einkommensverluste von Personen, deren Einnahmen von ebendiesen Betrieben abhängen, zu befürchten. ${ }^{14}$ Davon ausgehend, dass der durchschnittliche Anteil der Mietkosten am Haushaltseinkommen wie im Jahre 2017 ungefähr $30 \%$ beträgt, ${ }^{15}$ bedarf es keiner besonderen Phantasie um sich vorzustellen, dass starke Einkommensrückgänge eine nicht unerheblichen Zahl von Mietern schnell vor Schwierigkeiten bei der Mietzahlung stellen können. Was für die Wohnraummiete gilt, gilt erst Recht für die gewerbliche Miete: Brechen kurzfristig (wirtschaftsbedingt) die Aufträge bzw. (krankheitsbedingt) die Mitarbeiter weg oder muss der Betrieb gar wegen behördlicher Anordnung geschlossen bleiben, kann die Pflicht zur Entrichtung der Miete insbesondere bei kleinen Betrieben schnell zur existentiellen Belastung führen.

Unter Berücksichtigung der insofern „geringen“ Anforderungen an die Zahlungsverzugskündigung drohte die wirtschaftliche Krise mit einiger Verzögerung durch das Mietrecht verstärkt zu werden. Schon jetzt wird von einem Massensterben der mittelständischen Betriebe ausgegangen. ${ }^{16}$ Die Regelungen zur Zahlungsverzugskündigung hätten dies wohl noch erheblich verschärft. Selbst wenn sich die wirtschaftliche Lage innerhalb der nächsten Monate wieder etwas beruhigen sollte, was derzeit noch nicht absehbar ist, wird es eine Weile dauern, bis sich die Wirtschaft vollständig von dem „Schock“17 der Corona-Pandemie erholt hat. Die Monate März, April und Mai werden für viele Betriebe zur Durststrecke. Und so können auch an sich gesunde Betriebe wegen der außergewöhnlichen Situation Probleme haben, in diesen Monaten ihren Pflichten aus dem Mietvertrag nachzukommen. Der Verlust der Betriebsräume wäre allerdings häufig selbst für vor der Krise gesunde Unternehmen der Todesstoß. Von der so-

14 BT-Drucks. 19/18110, S. 16.

15 BT-Drucks. 19/18110, S. 16.

16 www.rnd.de/politik/corona-krise-im-mittelstand-gibt-es-ein-massensterben-von-un ternehmen-der-mittelstandsverbund-im-interview-GVUTRDOKONEORAPG6R WZIXHENU.html (zuletzt abgerufen am 21.04.2020).

17 M.-P. Weller/M. Lieberknecht/V.Habrich, Virulente Leistungsstörungen - Auswirkungen der Corona-Krise auf die Vertragsdurchführung, NJW 2020, 1017 (1022). 
zialen Krise, wenn größere Teile der Bevölkerung ihre Wohnungen verlieren, ganz zu schweigen.

Die Möglichkeit des Vermieters selbst bei unverschuldeten Zahlungsschwierigkeiten bereits nach spätestens zwei Monaten wegen Zahlungsverzug zu kündigen, scheint mit Blick auf durch die Corona-Krise bedingte Zahlungsschwierigkeiten in der Tat nicht angemessen. Unter gewöhnlichen Umständen darf der Vermieter nach zwei Monaten Zahlungsausfall berechtigter Weise damit rechnen, dass der Mieter auch zukünftig Schwierigkeiten haben wird, seinen vertraglichen Pflichten nachzukommen. ${ }^{18}$ Dies ist indes mit Blick auf die besondere wirtschaftliche Lage bei von der COVID-19-Pandemie Betroffenen gerade nicht der Fall. Hier kommen schließlich mitunter auch Personen in Schwierigkeiten, die unter normalen Umständen keine Probleme mit der Mietzahlung haben - etwa grds. gesunde Mittelstandsbetriebe oder Freischaffende bzw. von Kurzarbeit betroffene Mieter.

\section{Bloße Verlagerung von Liquiditätsproblemen}

Durch die Regelung in Art. $240 \$ 2$ Abs. 1 EGBGB wird sowohl das außerordentliche Kündigungsrecht als auch das ordentliche Kündigungsrecht des Vermieters wegen Zahlungsverzug ausgeschlossen. ${ }^{19}$ Die Kündigung aus sonstigen Gründen - wie etwa Eigenbedarf - bleibt hingegen möglich (Art. $240 \rrbracket 2$ Abs. 1 S. 3). Nun mag man einwenden, dass die Regelung das Problem nur verlagert. Das trifft grds. in zweierlei Hinsicht zu: Der Anspruch des Vermieters auf die Miete wird durch die Regelung ja gerade nicht berührt. Lediglich die einschneidende Konsequenz der Zahlungsverzugskündigung entfällt. Der Mieter muss die Zahlung - im Übrigen inklusive Zinsen ${ }^{20}$ - also später nachholen. Selbst nach einer wirtschaftlichen Normalisierung mag das vielen Mietern nicht ohne weiteres leicht fallen. Denn ihr Ausfall in den Monaten der Krise wird in den überwiegenden Fällen wohl nicht durch spätere Mehreinnahmen vollständig ausgeglichen. Nur weil Restaurants in den letzten Wochen geschlossen waren, steht bspw. nicht zu erwarten, dass die Menschen das Geld, welches sie in dieser Zeit „gespart“ haben in der Zeit nach der Normalisierung zusätzlich für

18 Vgl. BVerfG NJW 1989, 1917 Rn. 15 ff.

19 BT-Drucks. 19/18110, S. 36.

20 Insofern kritisch, ob der Grundsatz „Geld hat man zu haben“ auch in der CoronaKrise gilt: U. Börstinghaus, Besonderheiten des Miet- und Wohnungseigentumsrechts (Fn. 13), S. 413. 
auswertiges Essen ausgeben werden. Letztlich wird den Betroffenen durch die Bestimmung in Art. $240 \$ 2$ Abs. 1 EGBGB nur Zeit erkauft.

Andererseits wirkt sich das Ausbleiben der Miete natürlich auch auf die finanzielle Situation der Vermieter aus. Insofern kann die Regelung zu finanziellen Engpässen auf Vermieterseite führen. Es wird bereits orakelt, Immobilienbesitzer drohe wegen den ausbleibenden Mieten der Kollaps. ${ }^{21}$ Dem könnte man entgegenhalten, dass es - jedenfalls mit Blick auf das Corona-Rettungsgesetz - für solche Mieter, die finanzielle Polster haben, schon alleine wegen der anfallenden Zinsen (nach $₫ 288$ BGB immerhin 5 bzw. 9 Prozentpunkte über dem Basiszinssatz) wirtschaftlich vernünftig wäre, ihre Miete weiterhin pünktlich zu zahlen. ${ }^{22}$ Nur diejenigen, denen die Mietzahlung schlicht unmöglich ist, so sollte man annehmen, würden die geschuldete (und auch einklagbare) ${ }^{23}$ Miete nicht leisten. Ein massenhaftes Ausbleiben von Mietzahlungen dürfte auf Grundlage des CoronaRettungsgesetzes also eigentlich nicht zu erwarten sein. Eine andere Frage ist freilich, ob Mieter unter bestimmten Umständen - nach den allgemeinen Regeln - evtl. von der Pflicht zur Entrichtung der Miete befreit sind (hierzu später unter C).

\section{Voraussetzungen des Kündigungsausschlusses}

Es ist jedenfalls festzuhalten, dass Art. $240 \$ 2$ Abs. 1 EGBGB Mieter nicht dazu berechtigt, ihre Miete zurückzubehalten. Indes, welche Konsequenzen müssen finanzstarke Unternehmer fürchten, wenn sie es unberechtigter Weise doch tun? Droht Ihnen dann, wie Frau Lambrecht meint, trotz der Regelung in Art. $240 \$ 2$ Abs. 1 EGBGB ggf. eine Zahlungsverzugskündigung oder beschränken sich die Konsequenzen auf die Pflicht zur Rückzahlung mit Zinsen und ggf. weiteren Schadensersatz?

21 www.manager-magazin.de/politik/artikel/corona-krise-handel-hotels-restaurants-n iemandzahlt-mehr-miete-a-1305 795.html (zuletzt abgerufen am 21.04.2020).

22 Vgl. M. Artz, Interview mit Spiegel-Online vom 27.3.2020, abrufbar unter: www.spiegel.de/wirtschaft/service/corona-notmassnahmen-fuer-mieter-paket-kann -verheerende-auswirkungen-haben-a-e58a256e-32d1-4fb6-ac09-d2ccbea0d507 (zuletzt abgerufen am 21.04.2020).

23 E. Lindner, Die Beschränkung der Kündigung von Miet- und Pachtverhältnissen anlässlich der Covid-19-Pandemie (Art. $240 \$ 2$ EGBGB), AnwZert MietR 6/2020 Nr. 2. 


\section{1. „Subjektive Unmöglichkeit“ der Zahlung}

Auch insoweit dürfte Frau Lambrecht Recht haben: Der Ausschluss des Rechts zur Zahlungsverzugskündigung gilt nach dem Wortlaut der Bestimmung nur, sofern der Mieter glaubhaft machen kann, dass die Nichtleistung auf den Auswirkungen der COVID-19-Pandemie beruht. Zwar soll nach der Gesetzesbegründung für die Glaubhaftmachung Nachweise über die Gewährung staatlicher Leistungen, Verdienstausfälle oder der Hinweis auf eine behördlich angeordnete Schließung reichen. ${ }^{24}$ Prima facie scheinen also bereits Verdienstausfälle oder die behördliche Schließung zur Folge haben, dass man gegen Zahlungsverzugskündigungen geschützt ist. Bei näherer Betrachtung ist dies allerdings nicht der Fall. Zunächst betrifft die Frage nach der Glaubhaftmachung grds. nicht die Voraussetzungen für die Anwendbarkeit der Regelung selbst, sondern lediglich die Beweisführung mit Blick auf das Beweismaß und die Beweismittel. ${ }^{25}$ Voraussetzung für den Ausschluss der Zahlungsverzugskündigung bleibt, dass die Nichtleistung ihre Ursache in der COVID-19-Pandemie hat. Die Nichtleistung hat ihre Ursache jedoch nur dann in der Pandemie, wenn der Mieter wegen der Krise tatsächlich nicht in der Lage war, Miete zu bezahlen. Gemeint ist insofern die subjektive Unmöglichkeit, weil dem Mieter die (liquiden) finanziellen Mittel fehlen, seiner Verpflichtung zur Mietzahlung nachzukommen. Nicht ausreichend ist, dass der Mieter von der Krise betroffen ist und sich deshalb - gleichsam als „Vorsichtsmaßnahme“ um sein Geld zusammen zu halten -26 entscheidet, die Miete lieber nicht zu zahlen. ${ }^{27}$ Dies folgt ebenfalls bereits aus der Gesetzesbegründung. Dort heißt es: „Die Regelung stellt eine zeitlich begrenzte Ausnahme von dem Grundsatz dar, dass eine Leistungsunfähigkeit aufgrund wirtschaftlicher Schwierigkeiten den Schuldner auch dann nicht von den Folgen des Ausbleibens der (rechtzeitigen) Leistung befreit, wenn sie auf unverschuldeter Ursache beruht" ${ }^{\text {"28. }}$. Mit Blick auf den Sinn und Zweck der Regelung, nämlich solche Mieter schützen, die krisenbedingt in akute Liquiditätsengpässe gera-

24 BT-Drucks. 19/18110, S. $36 \mathrm{f}$.

25 I. Saenger, in: Nomos Handkommentar ZPO, 8. Auflage, Baden-Baden 2019, \$ 294 Rn. 1.

26 So wohl aber die Beweggründe bei Adidas, vgl. www.manager-magazin.de/politik /artikel/corona-krise-handel-hotels-restaurants-niemandzahlt-mehr-miete-a-1305 7 95.html (zuletzt abgerufen am 21.04.2020).

27 A.A. wohl U. Börstinghaus, Besonderheiten des Miet- und Wohnungseigentumsrechts (Fn. 13), S. 415.

28 BT-Drucks. 19/18110, S. 36. 
ten sind, wird man aber wohl nicht so weit gehen können, von Mietern zunächst zu verlangen, nicht liquide Mittel - etwa Lebensversicherung, Aktiendepots oder Erbschmuck - zu versilbern. ${ }^{29}$ Insbesondere dort, wo dies nur mit hohen Verlusten möglich ist - etwa mit Blick auf die massiven Kurseinbrüche auf dem Aktienmarkt - wäre dies vor dem Hintergrund des Schutzzwecks von Art. $240 \$ 2$ EGBGB unverhältnismäßig.

\section{Keine Vorprozessuale Pflicht zur Glaubhaftmachung}

Der Mieter muss glaubhaft machen, dass es ihm wegen der Corona-Krise unmöglich war, die Miete zu zahlen (Art. $240 \rrbracket 2$ Abs. 1 S. 2 EGBGB). Hierfür kann er auf Nachweise über Verdienstausfälle oder Ähnliches zurückgreifen. Ebenso kommt eine Versicherung an Eides statt in Betracht. Die bloße Glaubhaftmachung, wirtschaftlich von der Corona-Krise betroffen zu sein, reicht hingegen nicht aus. In diesem Zusammenhang stellt sich allerdings die Frage, ob Art. $240 \$ 2$ Abs. 1 S. 2 EGBGB als Voraussetzung für den Kündigungsausschluss zu verstehen ist, oder es sich um eine prozessuale Bestimmung handelt. Muss der Mieter also dem Vermieter gegenüber nachweisen, dass er coronabedingt nicht zur Mietzahlung in der Lage war, um den Kündigungsschutz zu aktivieren oder ist der Mieter ipso iure vor einer Zahlungsverzugskündigung des Vermieters geschützt, wenn die Voraussetzungen des S. 1 vorliegen und muss dies lediglich im Gerichtsverfahren glaubhaft machen? Wortlaut und Gesetzesbegründung sprechen für letzteres. ${ }^{30}$ Glaubhaftmachung ist, wie bereits gesagt, ein Terminus technicus des Prozessrechts. Zudem heißt es in der Gesetzesbegründung: „Gemäß Satz 2 obliegt es dem Mieter den Zusammenhang [...] im Streitfall glaubhaft zu machen. “31 Die Glaubhaftmachung soll also nur für den Streitfall notwendig und nicht per se erforderlich sein. ${ }^{32}$ Letztlich muss also ein Richter es aufgrund der vorgelegten Nachweise bzw. der Versicherung an Eides statt für überwiegend wahrscheinlich halten, dass

29 So aber $A$. Walther, Interview in der Süddeutschen Zeitung Online vom 17.4.2020, abrufbar unter: www.sueddeutsche.de/geld/rechtsanwalt-ein-wahres-au slegungswirrwarr-1.4877906 (zuletzt abgerufen am 21.04.2020).

30 Wie hier auch U. Börstinghaus, Besonderheiten des Miet- und Wohnungseigentumsrechts (Fn. 13), S. 414.

31 BT-Drucks. 19/18110, S. 36.

32 Wie hier M. Artz, in: Schmidt, COVID 19 - Rechtsfragen zur Corona-Krise, 1. Aufl., München 2020, $\$ 3$ Rn. 43 . 
der Mieter keine finanziellen Mittel zur Zahlung der Miete hatte. ${ }^{33}$ Hält ein Richter es hingegen für wahrscheinlicher, dass ausreichend finanzielle Mittel existierten um der Pflicht zur Entrichtung der Miete nachzukommen, wird der Mieter nicht durch den Ausschluss der Zahlungsverzugskündigung geschützt. Verweigert der Mieter also die Mietzahlung, obwohl er zur Leistung im Stande wäre, droht ihm die Kündigung wegen Zahlungsverzug, selbst wenn er von der Corona-Krise wirtschaftlich betroffen war. ${ }^{34}$ Ein Unternehmen wie Adidas, mit Rücklagen von mehreren hundert Millionen Euro, dürfte also grds. nicht durch Art. $240 \rrbracket 2$ Abs. 1 EGBGB geschützt werden.

\section{Kein Kündigungsausschluss bei Zablungsverzug vor oder nach dem Krisenzeitraum}

Eine andere Frage ist indes, ob sich nur solche Mieter auf den Schutz des Corona-Rettungsgesetzes berufen dürfen, bei denen nicht bereits vor der Corona-Krise Zahlungsrückstände bestanden. Die Gesetzesformulierung scheint uneindeutig. ${ }^{35}$ Dafür spricht, dass der Vermieter „nicht allein aus dem Grund kündigen" können soll, dass der Mieter seine Miete im KrisenZeitraum nicht geleistet hat. Man wird die Formulierung wohl nur so verstehen können, dass Nichtleistung im Krisenzeitraum zusammen mit vorherigen Zahlungsrückständen die Zahlungsverzugskündigung nicht ausschließt. ${ }^{36}$ Denn wenn andere Kündigungsgründe - etwa Eigenbedarf einschlägig sind, bedarf es keines Rückgriffs auf den Zahlungsverzug im Krisen-Zeitraum, um eine Kündigung des Vermieters zu rechtfertigen. Andererseits heißt es in der Bestimmung, dass dem Mieter nicht wegen Zahlungsverzug im Krisenzeitraum gekündigt werden kann, wenn er die Miete „trotz Fälligkeit“ nicht leistet. Auch die offene Miete aus den Monaten vor dem Krisenzeitraum ist natürlich „fällig“. Allerdings spricht einiges dafür, dass es sich insoweit um ein redaktionelles Versehen handelt und es

33 H. Prütting, in: Münchener Kommentar zur ZPO, 5. Auflage, München 2016, $\$ 294 \mathrm{Rn} .2$.

34 Vgl. auch BT-Drucks. 19/18110, S. 36: „Beruht die Nichtleistung des Mieters auf anderen Gründen, zum Beispiel weil er zahlungsunwillig ist [...], ist die Kündigung hingegen nicht ausgeschlossen“.

35 A.A. E. Lindner, Beschränkungen der Kündigung (Fn. 23): die Ausnahmeregelung lasse „keinen Zweifel aufkommen“ und greife „nur wenn der Mieter im Zeitraum von vom 01.04.2020 bis 30.06.2020 fällige werdende Mieten nicht leistet“.

36 M. Artz, Interview mit Spiegel-Online (Fn. 22). 
heißen müsste „Der Vermieter kann [...] nicht alleine aus dem Grund kündigen, dass der Mieter im Zeitraum vom 1. April 2020 bis 30. Juni 2020 fällig werdende Miete nicht leistet, [...].“ Der Gesetzesbegründung nach soll die Regelung nur Zahlungsrückstände erfassen die „vom 1. April bis 30. Juni 2020 entstehen" 37 . Dies würde auch dem bereits zuvor erläuterten Sinn und Zweck des Art. $240 \$ 2$ Abs. 1 EGBGB entsprechen: Es geht in der Bestimmung darum grds. gesunden Unternehmen bzw. zahlungskräftigen Mietern nicht ihre Existenzgrundlage bzw. ihren Lebensmittelpunkt wegen eines krisenbedingten Liquiditätsengpasses zu nehmen. Kam es jedoch schon vor der Krise zum Zahlungsverzug, scheint die Annahme eines grds. gesunden Unternehmens bzw. zahlungskräftigen Mieters - und damit die besondere Schutzwürdigkeit - zweifelhaft. Andererseits könnte die Gesetzesbegründung gegen eine so strenge Interpretation des Art. $240 \$ 2$ Abs. 1 EGBGB sprechen. Dort heißt es nämlich, dass sich aus den krisenbedingten Zahlungsrückständen weder ein wichtiger Grund i.S.d. $\$ 543$ BGB noch ein berechtigtes Interesse i.S.d. $\$ 573$ BGB entnehmen lasse. ${ }^{38}$ Darin klingt an, dass Zahlungsrückstände im Krisenzeitraum, sofern sie durch die Pandemie verursacht wurden, bei der Beurteilung ob ein wichtiger Grund bzw. ein berechtigtes Interesse vorliegt, generell nicht zu berücksichtigen sind. Insofern könnte der Gesetzgeber durch die Verwendung des Wortes „alleine“ lediglich beabsichtigt haben, klarzustellen, dass Zahlungsverzugskündigungen nicht ausgeschlossen sind, wenn Zahlungsrückstände außerhalb des Krisenzeitraums bereits für sich genommen die erforderliche Höhe von $\$ 543$ bzw. $\$ 573$ BGB erfüllen. $\mathrm{Zu}$ diesem Ergebnis wäre man allerdings wohl zwanglos auch ohne das Wort „alleine“ im Gesetzestext gekommen. Wäre dies also die Absicht des Gesetzgebers gewesen, hätte er gut daran getan, es ausdrücklich in der Gesetzesbegründung darzulegen oder besser ganz auf die vermeintliche „Klarstellung“ zu verzichten. Die besseren Argumente dürften damit letztlich für ein enges Verständnis der Voraussetzungen von Art. $240 \$ 2$ Abs. 1 EGBGB sprechen. ${ }^{39}$ Jedenfalls besteht insofern erhebliche Rechtsunsicherheit.

37 BT-Drucks. 19/18110, S. 36.

38 BT-Drucks. 19/18110, S. 36.

39 So i.E. auch E. Lindner; Beschränkungen der Kündigung (Fn. 22); U. Börstinghaus, Besonderheiten des Miet- und Wohnungseigentumsrechts (Fn.13), S.413; M. Artz, COVID 19 (Fn. 32), $\$ 3$ Rn. 41. 


\section{Stichtagsregelung in Art. $240 \$ 2$ Abs. 4 EGBGB}

Ein weiteres Problem im Zusammenhang mit Art. $240 \$ 2$ EGBGB ist, wie der dortige Abs. 4 zu versehen ist. Die Bestimmung sieht vor, dass die Absätze 1 bis 3 nur bis zum 30. Juni 2022 anzuwenden sind. Klar scheint, dass der Vermieter wegen Art. $240 \$ 2$ Abs. 4 EGBGB jedenfalls dann wegen Zahlungsverzug im Krisenzeitraum (01.04.2020 - 30.06.2020) kündigen kann, wenn der Mieter die offene Miete nicht bis zum Stichtag (30.06.2022) bezahlt.

Allerdings könnte man, so die teilweise geäußerte Befürchtung, der Stichtagsregelung auch entnehmen, dass das Kündigungsrecht des Vermieters wegen Zahlungsverzug in der Krisenzeit mit Ablauf des 30.7.2022 auflebt, selbst wenn der Mieter die Zahlungen unterdessen nachgeholt hat. ${ }^{40}$ Das Problem stellt sich vor dem Hintergrund, der dogmatischen Wirkung des Art. $240 \$ 2$ Abs. 1 EGBGB, die teilweise so verstanden wird, dass das Zahlungsverzugskündigungsrecht des Vermieters zwar entsteht, der Vermieter aber an der Ausübung gehindert ist. ${ }^{41}$ Allgemein wird in Konstellationen, in denen ein ordentliches Verzugskündigungsrecht zunächst entstanden ist, aber seine Voraussetzungen durch Zahlung vor Ausübung wieder erloschen sind, diskutiert, ob das Kündigungsrecht auch nach der Ausgleichszahlung erhalten bleibt. ${ }^{42}$ Dieses Problem würde sich nach Ablauf des Stichtags zweifellos auch mit Blick auf Zahlungsrückstände aus dem Krisenzeitraum stellen, wenn man davon ausgeht, dass der Vermieter nach Art. $240 \$ 2$ Abs. 1 EGBGB bloß die Ausübung in der Zahlungsverzugskündigung gehindert ist. Denn dann würde das Ausübungshindernis mit Ablauf des Stichtags entfallen und stünde der Zahlungsverzugskündigung nicht mehr entgegen. Da das Kündigungsrecht aber bereits zum Zeitpunkt des ausreichenden Zahlungsverzugs entstanden ist, müsste man entscheiden, welche Wirkung die zwischenzeitliche Zahlung auf das Kündigungsrecht hätte.

Die Annahme eines "Wiederauflebens“ des Kündigungsrechts trotz Zahlung der Rückstände bis zum Stichtag wäre kein angemessenes Ergebnis und ist so wohl auch vom Gesetzgeber nicht gemeint. Die Regierungsbegründung stellt vielmehr klar, dass die Zahlungsverzugskündigung wegen ausbleibender Mietzahlung im Krisenzeitraum nur dann in Betracht kommt, wenn der Mieter die Zahlungsrückstände bis zum 30. Juni 2022

40 M. Artz, Interview mit Spiegel-Online (Fn. 22).

41 So etwa M. Artz, COVID 19 (Fn. 32), \$3 Rn. 37.

42 Vgl. H. Blank, in: Schmidt-Futterer, 14. Auflage, München 2019, Rn. 31. 
nicht ausgeglichen hat. ${ }^{43}$ Damit, so heißt es in der Regierungsbegründung weiter, hätten Mieter und Pächter über zwei Jahre Zeit, einen zur Kündigung berechtigenden Miet- oder Pachtrückstand auszugleichen, bevor der Vermieter auf Grund der ausstehenden Zahlung kündigen kann. ${ }^{44}$ Aus diesem Grund wird mitunter eine gesetzgeberische Klarstellung gefordert, dass eine Zahlungsverzugskündigung dann ausscheidet, wenn der Vermieter bis zum Stichtag hinsichtlich der Miete aus dem Krisenzeitraum befriedigt wurde. ${ }^{45}$

Die Meinung, dass Art. $240 \$ 2$ EGBGB lediglich eine Sperrwirkung mit Blick auf das entstandene Zahlungsverzugskündigungsrecht zur Folge hat, liegt insbesondere dann nahe, wenn man davon ausgeht, die Glaubhaftmachung nach Art. $240 \$ 2$ Abs. 1 S. 2 EGBGB sei eine Voraussetzung für die Entstehung des Kündigungsschutzes. Denn dann würde Art. $240 \rrbracket 2$ Abs. 1 EGBGB keine Schutzwirkung entfalten, solange der Mieter seiner Obliegenheit zur Glaubhaftmachung gegenüber dem Vermieter nicht nachgekommen ist. Es wäre hier sogar denkbar, dass der Vermieter bereits die Zahlungsverzugskündigung ausgesprochen hat und der Vermieter sich erst dann unter Glaubhaftmachung des Zusammenhangs zwischen Nichtleistung und Pandemie auf Art. $240 \$ 2$ EGBGB beruft, was die Frage aufwirft, ob Art. $240 \$ 2$ EGBGB dann überhaupt noch Schutz bietet. Jedenfalls die Gefahr eines Widerauflebens der Kündigung nach Ablauf des Stichtags trotz zwischenzeitlicher Zahlung würde hier besonders naheliegen. Indes, die Auffassung, dass es sich bei der Glaubhaftmachung um eine materielle Voraussetzung für den Schutz des Mieters handelt, wurde bereits oben verworfen.

Aber die Annahme, dass Art. $240 \rrbracket 2$ Abs. 1 EGBGB nur eine Sperrwirkung bzgl. der Ausübung eines gleichwohl entstandenen Zahlungsverzugskündigungsrechts entfaltet, überzeugt auch generell nicht. Vielmehr dürfte die Vorschrift bereits die Entstehung eines Zahlungsverzugskündigungsrechts verhindern. Zwar ist die Formulierung von Art. $240 \$ 2$ Abs. 1 S. 1 EGBGB insoweit offen („kann nicht kündigen“). Allerdings folgt aus der Gesetzesbegründung, dass der Gesetzgeber mit der Regelung bereits die Entstehung des Verzugskündigungsrechts verhindern wollte. Denn dort heißt es: „Mietrückstände [aus dem Krisenzeitraum] stellen weder einen wichtigen Grund zur außerordentlichen fristlosen Kündigung [...] dar noch folgt aus ihnen ein berechtigtes Interesse zur ordentlichen Kündi- 
gung “46. Der Gesetzgeber geht also davon aus, dass ein Zahlungsverzug im Krisenzeitraum bereits nicht geeignet ist, um damit die Voraussetzungen der Verzugskündigung erfüllt sind. Damit würde ein Kündigungsrecht aber gar nicht erst entstehen und nicht bloß gehemmt werden.

Dementsprechend bedarf es hier nach der hier vertretenen Auffassung auch keiner ausdrücklichen gesetzlichen Ergänzung, dass die Kündigung ausgeschlossen ist, wenn der Vermieter vor Ablauf des 30.07.2022 befriedigt wird. Denn entsteht das Zahlungsverzugskündigungsrecht wegen Art. $240 \rrbracket 2$ EGBGB bereits nicht, stellt sich auch die Frage nicht, wie es sich auf entstandene Gestaltungsrechte auswirkt, wenn die Voraussetzungen nachträglich wieder entfallen. Die teilweise geforderte Klarstellung birgt vielmehr die Gefahr, dass dies als Argument für die Auffassung, bei der Glaubhaftmachung handele es sich um eine materielle Voraussetzung des Kündigungsschutzes, herangezogen wird. Besser wäre deshalb ausdrücklich klarzustellen, dass unter den (alleinigen) Voraussetzungen von Art. $240 \$ 2$ Abs. 1 S. 1 EGBGB bereits kein Zahlungsverzugskündigungsrecht des Vermieters entsteht.

\section{Umgehungsgefahr durch „Kautionsauffüllungsanspruch“}

Auf eine möglicherweise dramatische Lücke des Schutzes für Mieter, die coronabedingt nicht zur Mietzahlung in der Lage sind, hat allerdings Lindner hingewiesen: ${ }^{47}$ Sofern der Mieter eine Kaution als Sicherheit geleistet hat, kann sich der Vermieter, wenn die Mietzahlungen ausbleiben, u.U. aus dieser Kaution befriedigen. Dann hat der Vermieter allerdings nach $\$ 240$ BGB einen Anspruch auf „Wiederauffüllung“ der Kaution. Kommt der Mieter seiner Pflicht zur Widerauffüllung nicht nach, könnte dies den Vermieter zur Kündigung berechtigen. ${ }^{48} \mathrm{Ob}$ die Rechtsprechung hier mit Blick auf den Sinn und Zweck des Art $240 \$ 2$ Abs. 1 S. 1 EGBGB (etwa mit Verweis auf $\$ 242 \mathrm{BGB}$ ) intervenieren würde, ${ }^{49}$ oder eine solche Kündigung wegen des Wortlauts von Art $240 \$ 2$ Abs. 1 S. 3 EGBGB („Sonstige Kündigungsgründe bleiben unberührt.") zulässt, scheint derzeit offen.

46 BT-Drucks. 19/18110, S. 36.

47 E. Lindner, Beschränkungen der Kündigung (Fn. 23).

48 Ausführlich E. Lindner, Beschränkungen der Kündigung (Fn. 23).

49 Dafür E. Streyl, in: Schmidt, COVID 19 - Rechtsfragen zur Corona-Krise, 1. Aufl., München 2020, \3 Rn. 105. 
Eine entsprechende „Reparatur“50 des Gesetzgebers dürfte insoweit dringend erforderlich sein.

\section{Zwischenfazit}

Mit Blick auf die Regelung im Corona-Rettungsgesetz lässt sich jedenfalls folgendes Zwischenfazit ziehen: Die Regelung in Art. $240 \$ 2$ EGBGB soll ermöglichen die Last der aktuellen Krise auf viele Schultern zu verteilen. An der Pflicht des Mieters zur Zahlung der Miete ändert die Regelung nichts. Ihr Anwendungsbereich beschränkt sich zudem auf Fälle, in denen dem Mieter krisenbedingt kurzfristig die finanziellen Mittel zur Entrichtung der Miete fehlen. Diesen Mietern gegenüber kann vorübergehend nicht wegen des Zahlungsverzugs gekündigt werden. Insofern müssen Vermieter die Zahlungsengpässe ihrer Mieter mit ihren eigenen Reserven abfedern. In diesem Zusammenhang sei auch darauf hingewiesen, dass Art. 5 $\$ 4$ Abs. 1 Nr. 2 des Corona-Rettungsgesetzes eine Ermächtigungsgrundlage für die Bundesregierung enthält, die Kündigungsbeschränkungen durch Rechtsverordnung mit Zustimmung des Bundesrates auch auf Zahlungsrückstände im Zeitraum von Juli bis einschließlich September 2020 zu erstrecken.

\section{Auswirkungen der „Corona-Krise" nach den allgemeinen Regeln des Zivilrechts}

\section{Anwendbarkeit der allgemeinen Regeln des Zivilrechts?}

Es stellt sich zudem die Frage, ob Mieter wegen der Corona-Krise - unabhängig von Art. $240 \$ 2$ EGBGB - unter Umständen nach dem allgemeinen Zivilrecht nicht oder zumindest nur eingeschränkt zur Zahlung der Miete verpflichtet sein könnten. Der Gesetzgeber scheint mit Blick auf das „Corona-Rettungsgesetz“ zwar davon ausgegangen zu sein, dass die betroffenen Mieter in den meisten Fällen nicht von der Pflicht zur Mietzahlung befreit sind. ${ }^{51}$ So wird die Notwendigkeit der Regelung in Art. $240 \$ 2$ Abs. 1 EGBGB darauf gestützt, dass eine große Anzahl von Personen wegen der Krise nicht in der Lage wäre weiterhin fristgerecht ihre Miete zu

50 E. Lindner, Beschränkungen der Kündigung (Fn. 23).

51 So auch T. Drygala, Corona und ausbleibende Gewerbemieten (Fn. 6). 
bezahlen. ${ }^{52}$ Andererseits steht die Regelung einem Rückgriff auf das allgemeine Recht aber auch nicht im Weg. ${ }^{53}$ Dem Wortlaut nach beschränkt sich der Regelungsbereich von Art. $240 \$ 2$ Abs. 1 EGBGB auf Konstellationen, in denen überhaupt eine Pflicht zur Entrichtung der Miete besteht. Auch steht die Gesetzesbegründung einem Wegfall der Mietzahlungspflicht nach dem allgemeinen Zivilrecht nicht entgegen - schließlich wird dort ausdrücklich auf die allgemeinen Regeln mit Blick auf die Leistungspflicht des Mieters Bezug genommen („Mieter erhalten kein Leistungsverweigerungsrecht nach der Grundregel des $₫ 1$. Sie bleiben damit nach allgemeinen Grundsätzen zur Leistung verpflichtet [... $]^{3)^{54}}$.

Nach Ansicht von Drygala könnte indes der Sinn und Zweck von Art. $240 \$ 2$ Abs. 1 EGBGB gegen einen Ausschluss der Mietzahlungspflicht nach den allgemeinen Bestimmungen sprechen. Nach dem Willen des Gesetzgebers, so Drygala, soll das Gesetz den Leistungsverkehr so weit wie möglich aufrechterhalten und der Kündigungsschutz deshalb die einzige Hilfe für den Mieter sein. ${ }^{55}$ Allerdings ist dieser Schluss nicht zwingend. Es stimmt zwar, dass der Gesetzgeber sich bewusst gegen ein allgemeines pandemiebedingtes Leistungsverweigerungsrecht für Mieter entschieden hat. Dies ist auch sicher deshalb geschehen, weil der Gesetzgeber eine generelle Überwälzung von pandemiebedingten wirtschaftlichen Einbußen von Mietern auf die Vermieter als nicht interessengerecht angesehen hat. Das bedeutet allerding nicht, dass der Sinn und Zweck der Regelung einem Entfallen bzw. einer Reduktion der Miete dort entgegensteht, wo dies bereits nach den allgemeinen Vorschriften der Fall wäre. Vielmehr wollte der Gesetzgeber lediglich nicht auch für die darüberhinausgehenden Fälle ein Leistungsverweigerungsrecht einführen. Wo eine Leistungsverweigerung oder -reduktion vor der Krise interessengerecht schien, dürfte sie es wohl nach Ansicht des Gesetzgebers auch in der Krise noch sein. Das Vermieter im Rahmen der Corona-Krise zulasten der Mieter besser gestellt werden sollten, lässt sich dem Gesetz oder der Gesetzesbegründung jedenfalls an keiner Stelle entnehmen.

Als „allgemeine Grundsätze“, die mit Blick auf die Corona-Krise zu einem Wegfall bzw. einer Reduktion der Miete führen könnten, werden insbesondere die Mietminderung nach $\$ 536$ Abs. 1 BGB, die Unmöglich-

52 BT-Drucks. 19/18110, S. 36.

53 So i.E. auch M.-P. Weller/C. Thomale, Gewerbemietrecht - Mietminderung in der Corona-Krise BB 2020, S. 962; a.A. wohl T. Drygala, Corona und ausbleibende Gewerbemieten (Fn. 6).

54 BT-Drucks. 19/18110, S. 36.

55 T. Drygala, Corona und ausbleibende Gewerbemieten (Fn. 6). 
keit nach $\$ \$ 275$, 326 BGB und die Störung der Geschäftsgrundlage nach $\$ 313$ BGB diskutiert.

\section{Mietminderung nach $\$ 536$ Abs. 1 BGB}

Nach $₫ 536$ Abs. 1 BGB ist die Miete gemindert, soweit während der Mietzeit ein Mangel der Mietsache entsteht, der ihre Tauglichkeit für den vertragsgemäßen Gebrauch beeinträchtigt. Ist die Tauglichkeit für den vertragsgemäßen Gebrauch vollständig aufgehoben, ist der Mieter von der Pflicht zur Mietzahlung in Gänze befreit.

Mit Blick auf die Wohnraummiete dürften allenfalls coronabedingte Mängel von geringerer wirtschaftlicher Tragweite in Betracht kommen. So kann etwa die Nutzungsuntersagung von zur Mietwohnung gehörenden Spielplätzen einen Mangel darstellen, der zu einer Minderung von ca. 5\% berechtigt. ${ }^{56}$ Die Annahme, dass Ausgangs- oder Kontaktsperren einen zur Minderung berechtigenden Mietmangel begründen scheint hingegen fernliegend. ${ }^{57}$

Von größerer Bedeutung könnte $₫ 536$ Abs. 1 BGB mit Blick auf die Gewerberaummiete sein. Hier wird insbesondere diskutiert, ob Schließungen infolge der Erlasse der Landesregierungen nach dem Infektionsschutzgesetz einen tauglichkeitsbeeinträchtigenden Mangel darstellen, der nach $\$ 536$ Abs. 1 S. 1 BGB die Pflicht zur Mietzahlung entfallen lässt.

Drygala geht davon aus, dass die behördliche Schließung von Geschäften einen solchen Mangel darstellen kann. ${ }^{58}$ Er argumentiert, es würde sich dabei um einen „Umfeldmangel“ - also eine unmittelbare auf den Gebrauchswert einwirkende äußere Beeinträchtigung der Mietsache - handeln. Denn auch eine behördliche Maßnahme könne einen Umfeldmangel begründen, sofern sie sich unmittelbar gegen den Betrieb und nicht gegen die Person des Mieters richte. Dabei beruft er sich auf ein Urteil des XII. Zivilsenats des BGH vom 13.7.2011.59 Schaut man sich indes das genannte

56 K. Lützenkirchen, Auswirkungen der Corona-Pandemie (Fn. 12), S. 3; so auch Corona-Infoblatt des Berliner Mietervereins, S. 3, abrufbar unter: https://www.ber liner-mieterverein.de/downloads/bmv-corona-infoblatt-105.pdf.

57 K. Lützenkirchen, Auswirkungen der Corona-Pandemie (Fn. 12), S. 3; vgl. auch das Corona-Infoblatt des Berliner Mietervereins (Fn. 56) S. 1; E. Streyl, COVID 19 (Fn. 49), $₫ 3$ Rn. 47.

58 T. Drygala, Corona und ausbleibende Gewerbemieten (Fn 6).

59 BGH NJW 2011, 3151. 
Urteil an, lässt sich die Auffassung Drygalas jedoch nicht durch die BGHEntscheidung stützen.

In dem Urteil heißt es ausdrücklich: „Öffentlich-rechtliche Gebrauchshindernisse und Gebrauchsbeschränkungen, die dem vertragsgemäßen Gebrauch [...] entgegenstehen, begründen nach der Rechtsprechung des BGH [...] nur dann einen Sachmangel i.S. der $\$ \mathbb{S} 536 \mathrm{ff}$. BGB, wenn sie auf der konkreten Beschaffenheit der [Sache] beruhen und nicht in persönlichen oder betrieblichen Umständen des Pächters ihre Ursache haben."60 Und weiter heißt es dort: „Ergeben sich auf Grund von gesetzgeberischen Maßnahmen während eines laufenden Pachtverhältnisses Beeinträchtigungen des vertragsgemäßen Gebrauchs eines gewerblichen Pachtobjekts, kann dies nachträglich einen Mangel [...] begründen. Voraussetzung hierfür ist jedoch, dass die durch die gesetzgeberische Maßnahme bewirkte Gebrauchsbeschränkung unmittelbar mit der konkreten Beschaffenheit, dem Zustand oder der Lage des Pachtobjekts in Zusammenhang steht. Andere gesetzgeberische Maßnahme, die den geschäftlichen Erfolg beeinträchtigen, fallen dagegen in den Risikobereich des Pächters. “61

Die BGH-Entscheidung erging im Zusammenhang mit dem gesetzlichen Rauchverhobt durch das Nichtraucherschutzgesetz RheinlandPfalz. ${ }^{62}$ Dort ist eine Ausnahmeregelung für Gaststätten vorgesehen, die bestimmten baulichen Anforderungen genügen. ${ }^{63}$ Obwohl diese baulichen Anforderungen mit Blick auf die Vertragssache im entschiedenen Fall hätten hergestellt werden können, hat der BGH einen Minderungsanspruch des Pächters abgelehnt, weil die Gebrauchsbeeinträchtigung ihre Ursache nicht in dem Zustand oder der Beschaffenheit der Pachtsache hatte. ${ }^{64}$ Wenn aber ein zur Minderung berechtigender Mangel bereits in diesem Fall, in dem die bauliche Beschaffenheit des Vertragsgegenstands zumindest eine Rolle für die Nutzung gespielt hat, vom BGH abgelehnt wurde, dann lässt sich kaum behaupten, dass Mieter deren Geschäfte wegen der Corona-Erlasse geschlossen wurden, unter Zugrundelegung dieser Rechtsprechung die Zahlung „Zurecht“ verweigern könnten. ${ }^{65}$

Schon die Annahme Drygalas, dass der BGH in dem Urteil danach differenziere, ob sich der staatliche Eingriff gegen den Betrieb des Geschäfts oder gegen die Person des Betreibers richte, triff nicht zu. Es kommt nach

60 BGH NJW 2011, 3151, Rn. 8.

61 BGH NJW 2011, 3151, Rn.9.

62 NRauchSchG RP, Gesetz vom 05.10.2007, GVBl. 2007, 188.

$63 \rrbracket 7$ Abs. 3 NRauchSchG RP.

64 BGH NJW 2011, 3151, Rn. 10.

65 So aber T. Drygala, Corona und ausbleibende Gewerbemieten (Fn. 6). 
der Entscheidung vielmehr darauf an, ob der staatliche Eingriff im Zusammenhang mit der Beschaffenheit, dem Zustand oder der Lage der Mietbzw. Pachtsache steht oder nicht. Mit der Beschaffenheit oder dem Zustand der Miet- bzw. Pachtsache steht eine coronabedingte behördliche Geschäftsschließung in keinem Zusammenhang. ${ }^{66}$ Und auch die Argumentation, die coronabedingten Geschäftsschließungen stehen mit der Lage der Miet- bzw. Pachtsache im Zusammenhang, weil die Sache in Deutschland oder einen speziellen Bundesland belegen sei, und deshalb in den örtlichen Anwendungsbereich der Schließungserlasse falle, ${ }^{67}$ geht letztlich fehl. Dies folgt ebenfalls bereits aus der vorgenannten BGH-Entscheidung. Denn auch dort hätte man argumentieren können, dass die Lage der Pachtsache in Rheinland-Pfalz schließlich der Grund war, dass das dortige Nichtraucherschutzgesetz überhaupt anwendbar gewesen ist. Daraus, dass der BGH in seiner Entscheidung einen Mangel abgelehnt hat, ergibt sich, dass er den erforderlichen Zusammenhang zwischen behördlicher Einschränkung und der Belegenheit so nicht verstanden wissen wollte.

Letztlich liegt aber auch unabhängig davon in den coronabedingten behördlichen Nutzungsbeschränkungen kein „Umfeldmangel“ im Sinne der Rechtsprechung vor. Umfeldmängel beziehen sich auf Umstände, „die von außen auf die Mietsache unmittelbar einwirken" ${ }^{68}$. Die Nutzungsbeschränkungen wirken allerdings nicht unmittelbar auf die Mietsache ein. Denn (und das erkennt auch Drygala an) die Schließungsanordnungen beziehen sich nicht auf das Mietobjekt, sondern auf den verfolgten Geschäftszweck ${ }^{69}$ - können doch Lebensmittelgeschäfte, Bankfilialen Drogerien, Reinigungen und andere Geschäfte meist geöffnet bleiben. ${ }^{70}$ Insofern zutreffend stellt Streyl fest, dass der Vermieter nicht die Überlassung des Betriebs schuldet, sondern der dazu notwendigen Räume, sodass auch nur rechtliche Umstände, die die körperliche Beschaffenheit der Mietsache betreffen oder die Einfluss auf sie haben, den Leistungserfolg des Vermieters betref-

66 T. Drygala, Corona und ausbleibende Gewerbemieten (Fn. 6).

67 So wohl T. Drygala, Corona und ausbleibende Gewerbemieten (Fn. 6).

68 BGH NJW 2013, 680 Rn. 8.

69 T. Drygala, Corona und ausbleibende Gewerbemieten (Fn. 6).

70 Vgl. für NRW etwa www.land.nrw/de/wichtige-fragen-und-antworten-zum-coron a-virus? from $=$ mid1 \#0a57cfbb (zuletzt abgerufen am 21.04.2020). 
fen. ${ }^{71}$ Das ist mit Blick auf die Beschränkungen durch die Corona-Erlasse grds. jedoch nicht der Fall. ${ }^{72}$

Anders als Drygala geht Lützenkirchen davon aus, dass die coronabedingten behördlichen Nutzungsbeschränkungen keinen Mangel der Gewerberäume i.S.d. $\$ 536$ Abs. 1 BGB darstellen. ${ }^{73}$ Auch er geht dabei im Ausgangspunkt davon aus, dass die Einschränkung der Nutzbarkeit infolge behördlichen Einschreitens zu einem Mangel führen kann. ${ }^{74}$ Der Umstand, dass ein Mieter sein Geschäft nicht mehr oder nur noch eingeschränkt öffnen darf, könne die Voraussetzungen eines „sog. öffentlich-rechtlichen Mangels" erfüllen. ${ }^{75}$ Indes scheitere die Annahme eines zur Minderung berechtigenden Mangels nach Lützenkirchen letztlich daran, dass die Gebrauchsbeeinträchtigung nicht aus dem Risikobereich des Vermieters, sondern aus dem Risikobereich des Mieters stamme. ${ }^{76}$ Denn der Vermieter trage nur das Risiko für den baulichen Zustand, während der Mieter das Verwendungsrisiko trage, zu dem das Risiko mit dem Mietobjekt Gewinne erzielen zu können, gehöre. ${ }^{77}$ Im Ergebnis dürfte Lützenkirchen zuzustimmen sein: Die coronabedingten behördlichen Nutzungsbeschränkungen begründen keinen Mangel i.S.d. $\$ 536$ Abs. 1 BGB. ${ }^{78}$ Zwar ist auch Lützenkirchens Definition des öffentlich-rechtlichen Mangels nicht von den Urteilen gedeckt, auf die er diesbezüglich verweist. ${ }^{79}$ Aber in diesen Entscheidungen wird der Mangel wegen öffentlich-rechtlicher Gebrauchsbeeinträchtigungen ebenfalls ausdrücklich auf solche Fälle beschränkt, in denen die Beeinträchtigungen auf der Beschaffenheit der Sache beruht. ${ }^{80}$ Dies entspricht auch der ständigen Rechtsprechung des BGH. ${ }^{81}$ Und mit Blick

71 E. Streyl, COVID 19 (Fn. 49), $₫ 3$ Rn. 68.

72 Das gilt im Übrigen sowohl für die Lage vor dem 20.4.2020, als auch für die seit diesem Tag geltenden Beschränkungen für Geschäfte mit über 800qm Verkaufsfläche etwa nach der CoronaSchVO NRW in der bereinigten Fassung vom 17.04.2020, abrufbar unter: www.mags.nrw/sites/default/files/asset/document/202 0-04-17_coronaschvo_in_der_ab_20.04.2020_geltenden_fassung.pdf (zuletzt abgerufen am 21.04.2020).

73 K. Lützenkirchen, Auswirkungen der Corona-Pandemie (Fn. 12), S. 1.

74 K. Lützenkirchen, Auswirkungen der Corona-Pandemie (Fn. 12), S. 1.

75 K. Lützenkirchen, Auswirkungen der Corona-Pandemie (Fn. 12), S. 1.

76 K. Lützenkirchen, Auswirkungen der Corona-Pandemie (Fn. 12), S. 1.

77 K. Lützenkirchen, Auswirkungen der Corona-Pandemie (Fn. 12), S. 1.

78 So i.E. auch A. Schall, Corona-Krise: Unmöglichkeit und Wegfall der Geschäftsgrundlage bei gewerblichen Miet- und Pachtverträgen, JZ 2020, S. 388 (389).

79 Nämlich: BGH MDR 1980, 306; OLG Düsseldorf ZMR 2011, 685 und ZMR 1993, 275.

80 BGH MDR 1980, 306; OLG Düsseldorf ZMR 2011, 685.

81 Vgl. BGH BeckRS 1971, 31126177 I.2.a). 
auf die coronabedingten behördlichen Nutzungsbeschränkungen fehlt es wie soeben erläutert schlicht am Zusammenhang mit der Beschaffenheit der Geschäftsräume. ${ }^{82}$ Die betroffenen Mieter müssen ihre Geschäfte nicht deswegen geschlossen halten, weil eine Gefahr von den Räumen ausgeht, sondern weil eine Gefahr von den Menschen ausgeht, die sich andernfalls in den Geschäften aufhalten würden.

Zwar hat das RG mit Blick auf kriegsbedingte Verbote von Tanzveranstaltungen die Annahme eines Mangels bei einem gepachteten Tanzlokal in einem Urteil aus dem Jahr 1917 für „nicht zu beanstanden“ gehalten. ${ }^{83}$ Allerdings würde die Heranziehung dieser Rechtsprechung für die Vertragsparteien mit Blick auf die Corona-Krise im Ergebnis wohl Steine statt Brot bedeuten: In dem Verfahren hatte der Pächter auf Feststellung geklagt, dass er für die Dauer des Tanzverbots von der Verpflichtung zur Zahlung des Pachtzinses befreit sei. Der Verpächter hat widerklagend einen Räumungsanspruch geltend gemacht. Das RG kam zu dem Ergebnis, dass wohl beiden Ansprüchen stattgegeben werden könne. Aus dem Vertrag ergäbe sich, dass die verpachteten Räume die „Eigenschaften der Räume einer Tanzwirtschaft an sich tragen “. ${ }^{84}$ Das Tanzverbot hätte "die Ausnutzung dieser Eigenschaft verhindert, den Pachtgegenstand selbst getroffen und deshalb einen Mangel [...] verursacht". ${ }^{85}$ Jedoch, so das RG weiter, wäre das durch die Verbotsvorschrift geschaffene Erfüllungshindernis auch unter dem Gesichtspunkt der unverschuldeten nachträglichen Unmöglichkeit zu würdigen. ${ }^{86}$ Dies sei notwendig, sofern dem Verpächter kein vertragliches Kündigungsrecht zur Seite stehe und auch eine Zahlungsverzugskündigung mangels Zahlungspflichten des Pächters nicht in Betracht komme. ${ }^{87}$ Bei einem gegenseitigen Vertrag folge aus $\$ \$ 275$, 323 a.F. BGB (nunmehr $\$ 326$ BGB), dass der Schuldner bei einer von keiner Partei zu vertretenen Unmöglichkeit von seiner Leistungspflicht befreit werde und den Anspruch auf die Gegenleistung verliere. ${ }^{88}$ Diese Grundsätze können neben den Sondervorschriften der $\$ \mathbb{S} 536 \mathrm{ff}$. BGB zur Anwendung kommen, denn bei einem unverschuldeten unheilbaren Mangel komme weder ein Erfüllungsanspruch nach $\$ 536$ BGB a.F. (heute $\$ 535$ Abs. 1 S. 2 BGB) noch ein Schadensersatzanspruch des Mieters nach

82 So i.E. auch E. Streyl, COVID 19 (Fn. 49), $\$ 3$ Rn. 68 ff.

83 RGZ 89, 203.

84 RGZ 89, 203 (205).

85 RGZ 89, 203 (205).

86 RGZ 89, 203 (205).

87 RGZ 89, 203 (205).

88 RGZ 89, 203 (205). 
$\llbracket 538$ BGB a.F (heute $₫ 536$ a Abs. 1 BGB) in Betracht und $₫ 537$ BGB a.F. (heute $\$ 536 \mathrm{BGB}$ ) sehe eine mit den Rechtsfolgen des $\$ 323 \mathrm{BGB}$ a.F. (heute $\$ 326 \mathrm{BGB}$ ) übereinstimmende Regelung vor, sodass das Mietrecht keine besonderen Wertungen enthalte, die einer Anwendung von $\$ \$ \$ 275,323$ BGB a.F. (heute $\$ \mathbb{S} 275,326 \mathrm{BGB}$ ) entgegenstünden. ${ }^{89}$ Auch wenn es sich um eine bloße zeitweise Unmöglichkeit handele, könne diese, insbesondere wenn nicht absehbar sei, wann das Verbot wieder aufgehoben werde, einer endgültigen Unmöglichkeit gleichstehen. ${ }^{90}$ Deshalb könne in solchen Fällen aus dem Gebot von Treu und Glauben folgen, dass dem Verpächter „die Aushaltung des Vertrags nicht anzusinnen ist“. ${ }^{91}$ Hierbei sei eine Abwägung der beiderseitigen Interessen vorzunehmen, in die man auch die Unsicherheit einbeziehen müsse, dass der Pächter nach Beendigung des Verbots seine wirtschaftliche Tätigkeit eventuell nicht mit einem Erfolg wieder aufnehmen könne, „die eine Erfüllung seiner Zahlungspflicht gegenüber dem [Verpächter] ermöglicht““.92

Letztlich dürfte diese Rechtsprechung unterdessen überholt sein und zur Lösung der Fragen im Zusammenhang mit der Corona-Pandemie keine Relevanz mehr haben. Nicht nur scheint die Annahme, dass durch allgemeine Nutzungsbeschränkungen der Vertragsgegenstand „selbst getroffen" sei, vor dem Hintergrund der neueren BGH-Rechtsprechung im vorgenannten Urteil zum Nichtraucherschutzgesetz ${ }^{93}$ fraglich. Zudem sind die vom RG angenommenen Rechtsfolgen nicht sachgerecht. Dies gilt insbesondere für die Annahme dass wegen unverschuldeter vorübergehender nachträglicher Unmöglichkeit die beiderseitigen Leistungspflichten erlöschen und der Vermieter - sollte der Mieter nicht zur Zahlung der Miete bereit sein - Räumung verlangen können soll. Die Gefahr, dass die Corona-Krise für Mieter zum Verlust der Mieträume führen würde, sollte aus Sicht des Gesetzgebers schließlich gerade vermieden werden, weshalb des Art. $240 \$ 2$ EGBGB geschaffen wurde. Andererseits wird man sich aber auch nicht darauf beschränken können dem Urteil nur zu entnehmen, dass behördliche Schließungen einen Mangel der Miet- oder Pachtsache darstellen. Denn damit würde der im Urteil vorgenommene Interessenausgleich unzulässig beschränkt. Das RG hat erkannt, dass die Annahme eines Mangels im Interesse des Mieters einen Ausgleich für den Vermieter erfordert, der ebenso wenig für die Umstände verantwortlich ist.

89 RGZ 89, 203 (207).

90 RGZ 89, 203 (206).

91 RGZ 89, 203 (205f).

92 RGZ 89, 203 (206).

93 BGH NJW 2011, 3151. 
So sehr Überlegungen zum Schutz von Mietern, deren Geschäfte coronabedingt nur eingeschränkt oder gar nicht geöffnet sein können, auch nachvollziehbar sind - die Annahme eines die Tauglichkeit aufhebenden Mangels und die damit einhergehende Überwälzung der Folgen der Pandemie auf den Vermieter scheint genauso wenig gerecht, wie wenn die Mieter ihre Folgen alleine tragen muss. ${ }^{94}$ Letztlich dienen die beschlossenen Maßnahmen der Allgemeinheit, sodass es gerecht erscheint, die mit ihnen einhergehende Belastung auch auf möglichst viele Schultern zu verteilen. Im mietrechtlichen Mangelgewährleistungsrecht geht es um die $\mathrm{Zu}$ weisung von Gebrauchsbeeinträchtigungen nach Ursachensphären. ${ }^{95}$ Jedoch lässt sich eine Pandemie letztlich keiner Ursachensphäre der Vertragsparteien zurechnen, ${ }^{96}$ sodass das mietrechtliche Mangelgewährleistungsrecht letztlich nicht zu passen scheint. ${ }^{97}$

\section{Wegfall der Leistungspflicht nach $\int \mathbb{S} 326,275$ BGB}

In eine ähnliche Richtung wie die Entscheidung des RG geht die Ansicht von Schall, nach welcher die coronabedingten Schließungen zwar keinen Mangel der Mietsache begründen, aber zu einer Unmöglichkeit für den Vermieter führen, die den Mieter nach $\$ 326$ Abs. 1 S. 1 BGB von der Pflicht zur Mietzahlung befreit. ${ }^{98}$ Schall differenziert also zwischen der Nichterfüllung der vertraglichen Pflicht - diese sei mit Blick auf den Vermieter bei coronabedingten Geschäftsschließungen gegeben, weil sie in ihrer inhaltlichen Dimension auch die Art und Weise der vertraglichen Nutzung etwa als Geschäftsbetrieb umfasse - und einem Mangel - an diesem fehle es insoweit, weil die Nutzungsuntersagung ihren Grund nicht in der Mietsache habe. ${ }^{99}$ Auf den ersten Blick scheint die Differenzierung plausibel. Der BGH definiert den Mangel der Mietsache in ständiger Rechtsprechung schließlich als „für den Mieter nachteilige Abweichung

94 In diesem Sinne auch M.-P. Weller/M. Lieberknecht/V. Habrich, Virulente Leistungsstörungen (Fn. 17), S. 1021.

95 N. Eisenschmid, in: Schmidt-Futterer, Mietrecht, 14. Aufl., München 2019, $\$ 536$ Rn. $12 \mathrm{ff}$.

96 M.-P. Weller/M. Lieberknecht/V. Habrich, Virulente Leistungsstörungen (Fn. 17), S. 1021.

97 A.A. M.-P. Weller/C. Thomale, Mietminderung (Fn. 53), nach denen $₫ 536$ BGB mit Blick auf die coronabedingten Schließungen eine Minderung um 50\% zulässt.

98 A. Schall, Corona-Krise (Fn. 78), S. 389.

99 A. Schall, Corona-Krise (Fn. 78), S. 389. 
des tatsächlichen Zustands der Mietsache von dem vertraglich geschuldeten Zustand". ${ }^{100}$ Die Zustandsbezogenheit des Mangels scheint also Raum für Verletzungen der Hauptleistungspflichten des Vermieters zu lassen, die keinen Mangel i.S.d. $\$ 536$ BGB darstellen. Und in der Tat sind durchaus Fälle denkbar, in denen nach Gebrauchsüberlassung eine Unmöglichkeit vorliegt, aber kein Mangel - etwa die völlige nachträgliche Zerstörung der Mietsache. ${ }^{101}$

Bei näherer Betrachtung überzeugt die Differenzierung letztlich jedoch nicht. Einem Rückgriff auf $\mathbb{S} \$ 326,275$ BGB steht mit Blick auf die hier interessierenden Fälle entgegen, dass das mietrechtliche Gewährleistungsrecht insoweit das allgemeine Leistungsstörungsrecht verdrängt, ${ }^{102} \mathrm{da}$ andernfalls die Wertentscheidungen und Voraussetzungen der $\$ \$ 536 \mathrm{ff}$. BGB unterlaufen werden. ${ }^{103}$ Denn zwar mag das RG im oben genannten Urteil festgestellt haben, dass eine Anwendbarkeit des allgemeinen Leistungsstörungsrechts - nämlich $\$ \$ 275,326 \mathrm{BGB}$ - nicht ausgeschlossen sein muss, wenn man einen Mangel bejaht. ${ }^{104}$ Dabei hatte sich das RG allerdings auf die Erkenntnis gestützt, dass die Ergebnisse des allgemeinen Schuldrechts im konkreten Fall im Wesentlichen mit denjenigen des besonderen Mangelgewährleistungsrechts übereinstimmten. ${ }^{105}$

Geht man - entgegen der hier und auch von Schall vertretenen Ansicht - von einem Mangel der Mietsache wegen der Corona-Erlasse aus, wäre ein Rückgriff auf die allgemeinen Vorschriften aber gar nicht notwendig, um zu dem Ergebnis zu kommen, dass die betroffenen Mieter nicht zur Zahlung der Miete verpflichtet sind. Dies würde sich zwanglos aus $\$ 536$ Abs. 1 S. 1 BGB ergeben. Indes, wenn man einen Mangel ablehnt, würde man nach Schalls Ansicht über das allgemeine Leistungsstörungsrecht zu Ergebnissen kommen, die das besondere Mangelgewährleistungsrecht gerade nicht vorsieht und somit die Wertungen der $\$ \$ 536 \mathrm{ff}$. BGB unterlaufen.

Es lässt sich dabei auch nicht behaupten, dass die Fälle der coronabedingten Schließungen außerhalb des Anwendungsbereichs des besonderen mietrechtlichen Mangelgewährleistungsrechts liegen. Denn der Vermieter ist nach $₫ 535$ Abs. 1 S. 1 und 2 BGB verpflichtet dem Mieter den Gebrauch der Mietsache zu gewähren und die Mietsache den gesamten Vertragszeit-

100 BGH NJW-RR 1991, 204.

101 M. Bieder, in: Beck-online.GROSSKOMMENTAR, München Stand 01.01.2020, $\$ 536$ BGB Rn. 9.

102 M. Bieder (Fn. 101), $\$ 536$ BGB Rn. 4; E. Streyl, COVID 19 (Fn. 49), $\ 3$ Rn. 58.

103 So i.E. auch M.-P. Weller/C. Thomale, Mietminderung (Fn. 53).

104 RGZ 89, 203.

105 RGZ 89, 203. 
raum über in einem hierfür geeigneten Zustand zu erhalten. Zum $\mathrm{Zu}$ stand der Mietsache zählen dabei neben ihrer physischen Beschaffenheit alle tatsächlichen und rechtlichen Verhältnisse, sofern diese mit der Sache zusammenhängen. ${ }^{106}$ Daraus folgt, dass der Vermieter seine Hauptleistungspflicht lediglich auf zwei Arten „nicht erfüllen“ kann - nämlich durch die Nichtüberlassung der Mietsache oder durch die Überlassung der Mietsache, ohne dass diese sich in einem für den vertragsgemäßen Gebrauch geeigneten Zustand befindet. Konstellationen, in denen eine „Nichterfüllung“ des Vermieters vorliegt, ohne dass von einem Mangel gesprochen werden kann, sind allerdings nur im ersten Fall denkbar. Denn allenfalls wenn der Vermieter dem Mieter die Mietsache von Anfang an nicht überlässt, oder wenn die Mietsache nachträglich vollständig zerstört wird, lässt sich vernünftiger Weise auch unter Beachtung des weiten $\mathrm{Zu}$ standsbegriffs nicht von einem „Mangel der Mietsache“ sprechen. ${ }^{107} \mathrm{Hat}$ der Vermieter die Mietsache jedoch überlassen und ist die Sache noch existent, kommt eine „Nichterfüllung“ des Vermieters nur in Betracht, soweit die Mietsache sich nicht in einem für den vertragsgemäßen Gebrauch geeigneten Zustand befindet. Dies sind jedoch die klassischen Fälle des Mangels. Mit Blick auf die Beschränkungen durch die Corona-Erlasse bleibt also kein Raum für die Qualifikation als „Nichterfüllung“ durch den Vermieter ohne gleichzeitige Qualifikation als Mangel. Lehnt man jedoch einen Mangel $\mathrm{ab}$, muss man die Wertungen des $\$ 536$ BGB respektieren und kann nicht auf $\$ ₫ 326,275$ BGB zurückgreifen, um den Mieters seiner Leistungspllicht zu befreien.

Es lässt sich auch nicht argumentieren, dass die Bestimmungen des mietrechtlichen Mängelgewährleistungsrechts und die Bestimmungen der $\$ \mathbb{S} 326,275$ BGB unterschiedliche Risikoregeln betreffen und ihre Wertungen deshalb nicht miteinander kollidieren. Die Regelung in $\$ 536$ BGB ist unmittelbare Ausprägung des Äquivalenzprinzips und soll das Austauschverhältnis zwischen Leistung und Gegenleistung herstellen, soweit dieses wegen eines Mangels der Mietsache gestört ist. ${ }^{108}$ Bekommt der Mieter nicht was ihm versprochen wurde, so lässt sich der Regelungsgehalt zusammenfassen, muss er sich seinerseits auch nicht an sein Versprechen halten. Die Anpassung erfolgt deshalb ipso iure und es ist unerheblich, ob der Vermieter die Gebrauchsbeeinträchtigung zu vertreten hat oder nicht. Auch $₫ 326$ BGB ist Ausdruck der Verknüpfung von Leistungspflicht und 
Gegenleistungspflicht sowie des vertraglich vereinbarten Austauschverhältnisses. ${ }^{109}$ Der Regelung in $\$ 326$ Abs. 1 BGB liegt also ebenfalls die Überlegung zugrunde, dass der Schuldner nicht an sein Versprechen gebunden sein soll, wenn er nicht bekommt, was ihm versprochen wurde. ${ }^{110}$ Auch bei $\$ 326$ Abs. 1 BGB ist die Wirkung deshalb ipso iure und unabhängig vom Verschulden des Gläubigers. Damit geht es sowohl bei $₫ 536 \mathrm{BGB}$ als auch bei $\$ 326$ BGB um die Verteilung der „Preisgefahr“. Wenn das besondere mietrechtliche Gewährleistungsrecht dem Vermieter die „Preisgefahr“ für bestimmte Umstände nicht zuweist, würde ein Rückgriff auf das allgemeine Schuldrecht diese Wertung unterlaufen, wenn hierdurch die Preisgefahr für diese Umstände letztlich doch auf den Vermieter übergewälzt wird.

Zudem stellt sich bei einem Rückgriff auf $\$ \$$ 326, 275 BGB die Frage, ob der Vermieter - wenn ihm vorgehalten wird, er habe keinen Anspruch auf die Miete weil er seine vertraglichen Leistungspflichten nicht erfüllen würde - nicht auch Herausgabe der Räume, mithin die Räumung der Mietsache, verlangen können müsse. Dass der Mieter weiterhin im Besitz der Mietsache bleiben darf, aber der Vermieter keine Gegenleistung für die Besitzüberlassung bekommt, scheint jedenfalls kein überzeugendes Ergebnis. ${ }^{111}$ Dies entspricht auch der Auffassung des RG im oben genannten Urteil, das dem Vermieter die Überlassung der Mietsache ohne Anspruch auf die Miete für nicht zumutbar gehalten hat. ${ }^{112}$ Wenn dies allerdings mit Blick auf die mangelhafte Leistung des Vermieters gilt, so wird dies es erst Recht dann gelten müssen, wenn man - ohne einen Mangel zu bejahen die Leistungspflicht des Mieters unter Rückgriff auf das allgemeine Leistungsstörungsrecht herleitet.

109 C. Herresthal, in: Beck-online.GROSSKOMMENTAR, München Stand 01.06.2019, $\$ 326$ BGB Rn. 2.

110 J. D. Brinkmann, Rücktritt und verbraucherschützender Widerruf, Berlin 2018, S. 176.

111 Hier käme allerdings eine Lösung über $₫ 313$ BGB in Betracht - der Umweg über $\int \$ 326,275$ BGB zu $\$ 313$ BGB scheint insofern allerdings überflüssig und eine unmittelbare Anwendung von $\$ 313$ BGB näherliegend, hierzu sogleich, unter C.IV.

112 RGZ 89, 203. 


\section{Störung der Geschäftsgrundlage nach $\$ 313$ BGB}

Im Gegensatz zu der Gesetzeslage im Jahre 1917 bedarf es nach der heutigen Rechtsordnung mit Blick auf die coronabedingten Beschränkungen aber auch keines Rückgriffs auf die $\mathbb{S} 536 \mathrm{ff}$. und 326, 275 BGB. Denn gerade aus der Auseinandersetzung mit der Rechtsprechung des Reichsgerichts wegen der Folgen des ersten Weltkriegs wurde von Paul Oertmann die Theorie zur Störung der Geschäftsgrundlage entwickelt, welche sich im Laufe der Zeit zu einem bewährten Rechtsinstitut entwickelte und $2002 \mathrm{im}$ Rahmen der Schuldrechtsmodernisierung in $\$ 313$ BGB in das Gesetz eingeführt wurde. ${ }^{113}$ Insofern scheint diese Bestimmung geradezu prädestiniert für die Lösung des Interessenkonflikts zwischen Mietern und Vermietern wegen der coronabedingten behördlichen Beschränkungen. Dies gilt auch vor dem Hintergrund, dass die Vertragsaufhebung dort nur als ultima ratio vorgesehen ist und primär eine Vertragsanpassung erfolgt. So lässt $₫ 313$ BGB genug Spielraum, damit nicht einer Partei des Mietverhältnisses die Lasten der Corona-Krise alleine auferlegt werden, sondern eine faire Lastenverteilung möglich ist. ${ }^{114} \mathrm{Da}$ der Wegfall der Geschäftsgrundlage im Rahmen dieser Tagung bereits Gegenstand eines eigenen Vortrags ist, möchte ich mich diesbezüglich nur auf ein paar weitere Bemerkungen beschränken.

Sofern Lützenkirchen eine Anpassung der Miete nach $₫ 313$ BGB mit dem Argument ablehnt, dass das Verwendungsrisiko ausschließlich in den Bereich des Mieters falle, überzeugt dies nicht. Der Einwand von Lützenkirchen betrifft das sog. normative Element. Eine Vertragsanpassung nach $\$ 313$ BGB setzt insofern voraus, dass einem Teil unter Berücksichtigung aller Umstände des Einzelfalls, insbesondere der vertraglichen oder gesetzlichen Risikoverteilung, das Festhalten am unveränderten Vertrag nicht zugemutet werden kann. Anders formuliert, kann eine Partei keine Vertragsanpassung verlangen, wenn ihr das jeweilige Risiko, welches sich bei der Änderung der Umstände verwirklicht hat, vertraglich oder gesetzlich zugewiesen ist. ${ }^{115}$ Tatsächlich ist das Risiko, dass die Miet- bzw. Pachtsache wie geplant verwendet werden kann und dass sich bei gewerblichen Mietobjekten ein Gewinn erzielen lässt, grds. dem Mieter zugewiesen. ${ }^{116}$ Diese

113 S. Martens, in: Beck-online.GROSSKOMMENTAR, München Stand 01.04.2020, $\$ 313$ BGB Rn. 14, 16.

114 Ausführlich zur Anwendbarkeit und den Rechtsfolgen des $₫ 313$ BGB mit Blick auf die Corona-Pandemie: A. Schall, Corona-Krise (Fn. 78), S. 389 ff.

115 S. Martens (Fn. 113), $\$ 313$ BGB Rn. 50.

116 S. Martens (Fn. 113), $\$ 313$ BGB Rn. 74. 
Risikozuweisung ist indes nur mit Blick auf „normale“ wirtschaftliche Verhältnisse gedacht. ${ }^{117}$ Die Corona-Krise allerdings betrifft die sog. "große Geschäftsgrundlage"118 das Grundvertrauen in den Bestand der wirtschaftlichen und sozialen Rahmenbedingungen. ${ }^{119}$ Derart fundamentale Änderungen dürften im Rahmen der Zuweisung des Verwendungs- und Gewinnrisikos zum Mieter nicht erfasst sein. Für eine Vertragsanpassung nach $₫ 313$ BGB spricht m.E. zweierlei: Zunächst der Umstand, dass die coronabedingten Einschränkungen im Interesse der Allgemeinheit keiner der Parteien zuzurechnen ist, sodass insofern durchaus von einer „Gefahrengemeinschaft" ${ }^{120}$ gesprochen werden kann. Zum anderen ist zu berücksichtigen, dass die überwiegende Anzahl anderer potentieller Mieter/Pächter der fraglichen Gewerberäume insofern mit denselben Beschränkungen konfrontiert wären, wie der konkrete Mieter. Insofern dürfte der wirtschaftliche Wert von Räumen zum Betrieb von Läden, Gaststätten, Diskotheken etc. unter Berücksichtigung von Angebot und Nachfrage derzeit sehr begrenzt sein. ${ }^{121}$ Aus diesem Grund ist davon auszugehen, dass ein redlicher Vermieter/Verpächter sich, hätte man die gegenwärtige Situation bei Vertragsschluss vorausgesehen, auf eine entsprechende Preisanpassungsklausel eingelassen hätte.

\section{Fazit}

Zusammenfassend lässt sich also festhalten, dass es wohl 2:0 für Frau Lambrecht steht. Weder das Corona-Rettungsgesetz noch die allgemeinen Regelungen des BGB berechtigen Mieter dazu ihre Miete wegen der CoronaKrise nicht zu zahlen. Insbesondere begründen die Corona-Erlasse der Länder grds. keinen Mangel der Mietsache. Allenfalls ein Anspruch auf Anpassung der Miete nach $₫ 313$ BGB dürfte insofern in Betracht zu kommen.

117 Vgl. P. Krebs/S. Jung, in: Nomos Kommentar BGB, 3. Aufl., Baden-Baden 2016, $\$ 313$ BGB Rn. 70; E. Streyl, COVID 19 (Fn. 49), 33 Rn. 81.

118 T. Finkenauer, in: Münchener Kommentar zum BGB, 8. Aufl., München 2019, $\$ 313$ BGB Rn. 17.

119 M.-P. Weller/M. Lieberknecht/V. Habrich, Virulente Leistungsstörungen (Fn. 17), S. 1021.

120 T. Finkenauer (Fn. 118), $\$ 313$ Rn. 306.

121 Wie hier E. Streyl, COVID 19 (Fn. 49), \$3 Rn. 86. 